\title{
A Heuristic Group Intelligence Control System Based on Hybrid Particle Swarm Gravitational Search Algorithm
}

\author{
Linshan Ding, Zhengya Wang, Tao Hu, Yi Liu and Li Zhang* \\ Huazhong University of Science \& Technology, Address: Luoyu Road 1037, Wuhan, China \\ ${ }^{*}$ Corresponding author
}

\begin{abstract}
Cluster control system to realize mutual coordination between individual objects must determine the control and the information relationship in terms of logical and physical aspects. Study these problems of the system structure, system structure and control can be combined and ensure smooth information flow and control flow in the system and the framework for the interaction between the individual. Cluster control algorithm ensure cooperation among multiple control individual effectively, to deal with an emergency to be able to react quickly, improve the real-time performance and effectiveness of control system. This paper proposed a new methodology to Group Intelligence Control by using a distributed control algorithm and a Swarm Intelligence algorithm to optimize the path of the individual of the cluster and the real-time control of each object. In the first part of this paper, we introduced the appearance and the development of Group Intelligence Control system. Then, the paper proposed our methodology of the proposed Group Intelligence Control system.
\end{abstract}

Keywords-cluster control; particle swarm optimization; gravitational search algorithm; distributed control algorithm; hybrid particle swarm gravitational search algorithm; tool-path optimization

\section{INTRODUCTION}

The purpose of Group Control System is to achieve mutual coordination between individuals, for which the controlling and information relationships of logical and physical of individuals must be determined[3]. The proposed system integrates the system structure and controlling to ensure the fluency of the information and controlling flow and to provide interactive framework for the communication of the individuals. Group Control System should guarantee the coordination of multiple individuals and real-time response of emergency.

Swarm Intelligence (SI) is the simulation of collective behavior of gregarious colony, which is a distributed, decentralized and self-organized system[4]. Swarm Intelligence can be used on robots or other control objects, in which each object has a fairly simple function, then after the individual is clustered, the function of the hole system becomes quite complex. The hole cluster can be considered as a distributed system including ground robot cluster, UAV cluster, underwater robot cluster etc, which compared with the individual, will improve fault redundancy.
The appearance of Swarm Intelligence promoted the development of Group Intelligent Control(GIC), in which Particle swarm optimization(PSO) algorithm and Gravitational search algorithm (GSA) applied to multiple mobile robot on holonomic wheeled platforms[11]. For example, the proposed hybrid particle swarm gravitational search algorithm which adopting co-evolutionary techniques to update the IGSA acceleration and particle positions with IPSO velocite simultaneously[5]. In the queue robot make decisions independently, through the coordination and cooperation between each other from the current position to determine their next position[12].

This paper proposed a new methodology to Group Intelligence Control by using a distributed control algorithm and a Swarm Intelligence algorithm to optimize the real-time control of each object[6]. In the first part of this paper, we introduced the appearance and the development of Group Intelligence Control system. Then, the paper proposed our methodology of the proposed Group Intelligence Control system.

The remaining part of the paper is outlined as follows. We explained the principle of the control system in Section 2, then the distributed controlling algorithm and the hybridization of GSA and PSO algorithm are given in Section 3 and Section 4. Next, we described the framework of our Group Intelligence Control system in Section 5. In section 6 experiment is conducted and finally, a conclusion of the work of this paper is presented.

\section{GROUP CONTROL SYSTEM}

To improve the real-time and effectiveness of the system, a typical control system is applicated, a STM32 series of microcontroller named STM32F405 is used as the main chip, which receives the control information of the host computer and turn it into the force which is needed in each object[7]. The control system uses PID algorithm, and make real-time measuring of the individuals, by comparing the supplied forces and the measurements, the system will do adjustment on each individual to assure that the hole cluster is regularly running. Meantime, the measurements are delivered to the host computer to make calculations to compute control information, which will be explained in the next part of the paper. Figure 1. shows the structure of the Group Control System. 


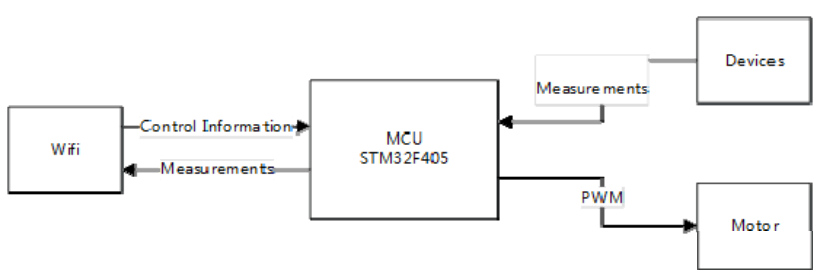

FIGURE I. STRUCTURE OF GROUP CONTROL SYSTEM

When measurements are received, the control system will deliver them to the host computer by wireless local area networking. After fast computing, the control information will be sent by host computing to the control system, by which realtime controlling will be achieved.

\section{Distributed CONTROL Algorithm}

The Distributed Control Algorithm is an individual based algorithm. The movement pattern of every individual is uncomplicated, when receiving the measurements delivered by the control system, this paper proposed a high-performance algorithm to determine the movement of each individual[2].

The steps of this algorithm are as follows:

Step 1. All the individuals are updated and measurements are delivered to the host computer.

Step 2. The first individual uses measurements and the previous states to calculate the difference considering the global path and send the difference to the second individual.

Step 3. The next individual will test the received difference, in most normal cases, the difference will be the same or similar so we do not need to recalculate it. If the test failed, it should have done another calculation of the difference using the measurements of the second individual. At last, send the difference to the next individual.

Step 4. For all individuals execute Step 3.

Step 5. Iterate Step 2 to Step 4 if needed.

The final states are the control information with the global path, and will be sent to the control system explained in Section 2 , finally, the information will be translated into forces that the individuals needed and delivered to each individual. The flow chart of Distributed Control Algorithm is presented in Figure 2.

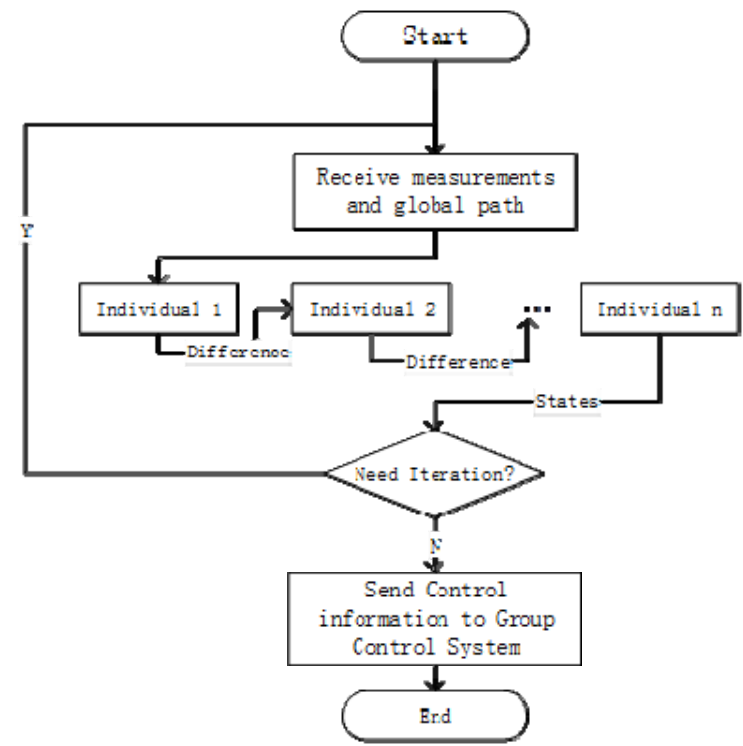

FIGURE II. FLOW CHART OF DISTRIBUTED CONTROL ALGORITHM

\section{HyBrid PARTICLE SWARM OPTIMIZATION AND GRAVITATIONAL SEARCH AlgORITHM}

In order to better solve the optimization problem, basically all the swarm intelligence algorithms such as particle swarm optimization (pso) algorithm and gravitational search algorithm are as far as possible to maintain the balance of the global search and local search[10]. In the particle swarm optimization (pso) algorithm, the individual extremum and global extremum respectively provide local search ability and global search ability. Have multidimensional space search ability of particle swarm optimization (pso), however in the local search ability of particle swarm optimization (pso) weaker than gravitational search algorithm.

Considering the advantages and disadvantages of particle swarm optimization (pso) algorithm and the advantages and disadvantages of gravitational search algorithm[9].The combination of particle swarm optimization (pso) algorithm and gravitational search algorithm has a variety of ways, such as using the output of particle swarm optimization (pso) or the gravitational search algorithm as the input of particle swarm optimization (pso) or the gravitational search algorithm. Due to the accuracy of the input value depends on the output value, so this kind of combination way short of the expected effect. This paper adopts a new way of the combination of particle swarm optimization (pso) algorithm and gravitational search algorithm, called the combining algorithm for the hybrid particle swarm gravitational search algorithm. In this algorithm, particles according to the speed of particle swarm optimization (pso) algorithm and the acceleration of gravitational search algorithm to update their location.

The speed of the hybrid particle swarm gravitational search algorithm position update formula is: 


$$
\begin{aligned}
& V_{1}^{\mathrm{d}}(\mathrm{t}+1)_{\mathrm{HPSO}-\mathrm{GSA}}=\mathrm{C}_{3} \varphi_{3} V_{1}^{\mathrm{d}}(t+1)_{\mathrm{PSO}}+\mathrm{C}_{4}(1- \\
& \left.\varphi_{3}\right) \mathrm{V}_{1}^{\mathrm{d}}(\mathrm{t}+1)_{\mathrm{GSA}}
\end{aligned}
$$

$$
x_{1}^{d}(t+1)=x_{1}^{d}(t)+V_{1}^{d}(t+1)_{\text {FPSO-GSA }}
$$

The $\mathrm{V}^{\mathrm{d}}(\mathrm{t}+1)_{\mathrm{pso}}$ from the particle swarm optimization algorithm update formula:

$$
\begin{aligned}
& V_{1}^{d}(t+1)_{P S O}-w V_{1}^{d d}+C_{1} \varphi_{1}\left(x_{p b e s t i}^{d}-x_{1}^{d}(t)\right)+ \\
& C_{2} \varphi_{2}\left(x_{\text {gbest }}^{d}-x_{1}^{d}(t)\right)
\end{aligned}
$$

$$
x_{1}^{d}(t+1)_{\text {PSO }}=x_{1}^{d}(t+1)+V_{1}^{d}(t+1)
$$

The $\mathrm{V}^{\mathrm{d}}(\mathrm{t}+1)$ GSA from the gravitational search algorithm update formula:

$$
V_{1}^{\mathrm{d}}(\mathrm{t}+1)_{G S A}=\beta V_{1}^{\mathrm{d}}(t)+a_{\varepsilon}^{d}(t)
$$

In the hybrid particle swarm gravitational search algorithm equation, $\mathrm{C}_{3}$ and $\mathrm{C}_{4}$ for two acceleration coefficient, used to adjust the speed of particle swarm optimization (pso) algorithm and the acceleration of gravitational search algorithm, $\Phi_{3}$ is any number of $[0,1]$, the size of its value to determine the speed of particle swarm and the acceleration of gravitational search algorithm proportion[1].

Hybrid algorithm process is as follows:

Step1: Set parameters, Generate the initial path populations.

Step2: According to the fitness function calculation the fitness value of each particle in the population.

Step3: Update the global extremum gbest, Individual extremum pbest, Inertia weight w.

Step4: Update the worst fitness function value worst and The best fitness function value best.

Step5: Calculate the gravitational constant during each iteration G, Every particle of inertial mass $M$ and the acceleration a.

Step6: Update each particle's speed and position.

Step7: To judge whether meet the termination conditions, meet the output the final result, does not meet the return to the step2.

\section{THE FRAMEWORK OF GROUP INTELLIGENCE CONTROL SYSTEM}

This paper uses hybridized PSO and GSA algorithm for path-planning optimization. The process is executed during issues when an individual has problems moving or an obstacle emerged in the calculated path[8]. At first the algorithm will calculate the global path before the system started and when the above issues occurred, the global path would be recalculated and be updated in the whole system. This algorithm will be combined with Distributed Control Algorithm given in Section 3 to compute a heuristic path, which will improve the fault tolerance of the cluster system. The framework of the system uses the above algorithms and system in Section 2, Section 3 and Section 4. The following part describes the process of the this methodology.

At first, the control system in Section 2 receive the measurements from all individuals, which are delivered to the host computer. Then the host computer will check the states of each individual and the whole system to decide if the global path is needed to recalculate, which uses the Algorithm in Section 4. After the global path is completed, the path and measurements will be used by Distributed Control Algorithm in Section 3. The entire system is integrated as a real-time Group Intelligence Control System, which is proposed by this paper. The framework of the proposed system is showed in Figure 3.

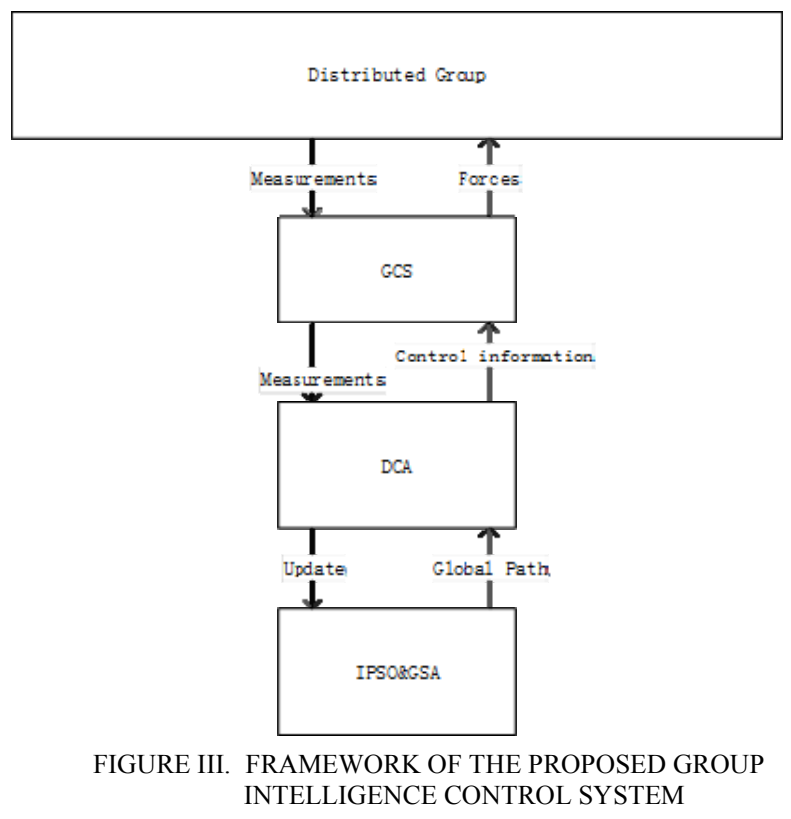

The algorithm of the system is as follows.

Step 1. The Group Control System get the measurements including the information of position, speed, angle, acceleration of each individual.

Step 2. The measurements will be sent to the host computer, the host computer will analyze the global path and each individual statement to decide whether the global path needs to be updated. If the global path needs to be updated, execute Step 3, else execute Step 4.

Step 3. The Hybridized IPSO and GSA algorithm receives the global statements and recalculate the global path considering the issues, after calculating, the global path is updated. 
Step 4. The Distributed Control Algorithm receives the measurements and global path, which are used to calculate the control information, and send the information to the Group Control System.

Step 5. The Group Control System receives the control information and translate it into forces which the individuals use, and send the forces to each individual.

\section{ACKNOWLEDGMENT}

This work was supported by the program of Piezoelectric Compound Vibration Energy with Pattern Gradation (0204100262).Thanks for the funds provided by associate professor li zhang and the working environment provided by Huazhong University of Science and Technology, School of Mechanical Science and Engineering.

\section{CONCLUSION}

This paper proposed a new methodology of group intelligence control system, for controlling the individuals of a cluster system effectively and accurately. The adjustment is made rapidly when emergency like collision and obstacle occurs. The proposed system will improve the mobility of individuals and the real-time of the system. An optimized global path will be provided by the algorithm and real-time controlling is achieved. Though the proposed system considered the movement path-planning of individuals and the collision, which is more applied to two-dimensional plane, the system is able to be updated in 3-dimentional space in the future.

\section{REFERENCES}

[1] P.K. Das , H.S. Behera , B.K. Panigrahi .A hybridization of an improved particle swarm optimization and gravitational search algorithm for multirobot path planning[J].Swarm and Evolutionary Computation 28(2016)14-28.

[2] Xiaogang Wang, Qin Wutao, Bai Yuliang, Naigang Cui. A novel decentralized relative navigation algorithm for spacecraft formation flying[J].Aerospace Science and Technology 48(2016)28-36

[3] Zhixiong Liu,Hua Liang. Particle swarm optimization (pso) algorithm in random number parameter setting and experimental analysis.[J]. Control theory and application ,2010,(11):1489-1496.

[4] Jianhua Liu. The basic theory of particle swarm algorithm and its improvement research[D].Central South University,2009.

[5] Moêz Soltani,Abdelkader Chaari,Fayçal Ben Hmida. A novel fuzzy cregression model algorithm using a new error measure and particle swarm optimization[J]. International Journal of Applied Mathematics and Computer Science,2012,22(3):.

[6] Jin Guofeng,Zhang Wei,Yang Zhengwei,Huang Zhiyong,Song Yuanjia,Wang Dongdong,Tian Gan. Image Segmentation of Thermal Waving Inspection based on Particle Swarm Optimization Fuzzy Clustering Algorithm[J]. Measurement Science Review,2012,12(6):.

[7] Xing Xu,Na Hu,Weiqin Ying, Yu Wu,Yang Zhou. The Particle Swarm Differential Evolution Algorithm for Ecological Sensor Network Coverage Optimization[J]. Journal of Intelligent Systems,2016,25(3):.

[8] Vimal Kumar Pathak,Amit Kumar Singh,Ramanpreet Singh,Himanshu Chaudhary. A modified algorithm of Particle Swarm Optimization for form error evaluation[J]. tm - Technisches Messen,2017,84(4):

[9] Weifeng Fan. Gravitational search algorithm analysis and improvement[D]. Guangdong university of technology,2014.
[10] Xiuling Zhang,Jiayin Zang,Hongmin Fan,Liang Zhao. The improved gravitational search algorithm[J]. Journal of shenyang university, 2014,(06):468-472.

[11] Meiyan An,Zhaokui Wang,Yulin Zhang. Artificial intelligence (ai) cluster control demonstration system[J]. Robot, 2016, (03) : 2016-275.

[12] Jiangzheng Sun,Chunxia Sun. Based on color recognition of smart car cluster control [J]. Journal of single-chip microcomputer and embedded system application, 2014, (12) : 45-48. 